\title{
Lifting off a solid sphere from a flat bottom by laminar fluid flow
}

Chao Wang ${ }^{\text {a,b,c }}$, Hanbin Wang ${ }^{\text {a,b }}$, Kun Zhang ${ }^{\text {a,b }}$, Zhipeng Li $^{\text {a,b, }}{ }^{,}$, Zhengming Gao ${ }^{\text {a,b, }}{ }^{,}$, J.J. Derksen ${ }^{\text {c }}$

${ }^{a}$ Beijing Advanced Innovation Center for Soft Matter Science and Engineering, Beijing University of Chemical Technology, Beijing 100029, China

${ }^{\mathrm{b}}$ State Key Laboratory of Chemical Resource Engineering, School of Chemical Engineering, Beijing University of Chemical Technology, Beijing 100029, China

${ }^{\mathrm{c}}$ School of Engineering, University of Aberdeen, Aberdeen, AB24 3UE, UK

Correspondence concerning this article should be addressed to Zhipeng Li at lizp@ mail.buct.edu.cn or Zhengming Gao at gaozm@buct.edu.cn

\begin{abstract}
We perform quantitative visualization experiments on the vertical ( $z$-direction) motion of a spherical solid particle being lifted off a horizontal flat bottom due to laminar fluid flow generated by a revolving impeller. Describing the observed motion of the particle in terms of a constant vertical hydrodynamic force overcoming gravity and the lubrication force has limited success. For this reason we hypothesize that the hydrodynamic force on the particle quickly increases with its distance from the bottom. This hypothesis is supported by detailed numerical simulations of the flow around the particle. Integrating the equation of motion of the sphere with the vertical hydrodynamic force as a linear function of $z$ derived from simulations provides an adequate description of the experimentally observed vertical motion of the particle.
\end{abstract}

\section{Keywords}

Solids suspension, quantitative visualization, lubrication force, particle-resolved simulation, agitated flow 


\section{Introduction}

Suspending solid particles in a liquid stream is at the heart of a number of applications in (chemical) engineering. It is a way of transporting large amounts of solids through pipeline systems as in dredging; it is a way of achieving mass transfer between solids and a liquid phase as in crystallization or dissolution processes, and in catalytic slurry reactors. Designing the particulates as well as the fluid flow so that particles stay suspended with minimum energy requirements is thus a relevant topic of research and development. In this respect it is important to describe and understand how a particle makes the transition from resting on a solid (bottom) surface to being entrained by fluid flow. This requires knowledge of the hydrodynamic forces - and possibly moments - on particles resting on, or closely above a solid surface.

There are many papers on spherical particle motion in the vicinity of a wall in a viscous fluid. Results have been summarized by Leal $^{1}$, Feuillebois ${ }^{2}$ and Michaelides ${ }^{3}$ in review papers. As for the translation of a sphere parallel to a smooth plane wall, Goldman et al. ${ }^{4}$ provided asymptotic lubrication solutions for the case such that the gap between the sphere and the plane approaches zero. Brenner ${ }^{5}$ pioneered an analytical approach for solving the quasi-steady Stokes equations for a sphere (with constant velocity) moving in the wallnormal direction towards or away from a plane wall in slow, viscous flow in the form of a series solution. In order to deal with divergence for small distances between sphere and wall, Cox and Brenner ${ }^{6}$ developed lubrication theory for the force on the sphere in this limiting case.

Incipient motion of solids and particle resuspension in liquid has also attracted extensive attention. Rabinovich and $\mathrm{Kalman}^{7}$ studied the incipient motion of particles experiencing shear flow in horizontal pipeline systems. The force and moment balances were used to obtain empirical models based on experimental results. The mechanisms of incipient 
motion for the single particle were determined by analyzing under what conditions encouraging forces (drag and lift forces) overcome resisting ones (gravity, adhesion and friction forces $)^{8}$. The motion of the particle starts by rolling, sliding or lifting. The rolling mechanism occurs once the moments due to drag and lift force overcome the resisting moments. If the drag force equals the friction force, the initial particle motion is sliding. The particle is lifted off the bottom wall when the lift force becomes equal to the sum of adhesive and gravity force in the vertical direction. Soepyan et al. ${ }^{9}$ have reviewed numerous studies on incipient motion as presented in the literature concerning the parameter ranges, forces, and mechanisms. The paper evaluated various models used in past research and suggested ways for their improvement. As for the studies of resuspension, mechanisms were given by coupling between three major physical interactions, that is, particle-fluid, particle-surface, and particle-particle interactions, which were summarized by Henry and Minier in their review paper ${ }^{10}$. Recently, by referring the published experimental data, several mechanistic models for particle-substrates/surfaces resuspension were introduced and validated by Nasr et al. ${ }^{11}$. The surface roughness and shape of particle have effect on the predictive capability of the model. Shnapp and Liberzon ${ }^{12}$ studied the effect of surface roughness on resuspension in a vortex-like flow.

The entrainment of solid particles in a stirred viscous flow is a common phenomenon in various engineering applications. Particles collect - due to settling - on the bottom of a container filled with liquid. Agitating the liquid with an impeller generates a fluid flow that once strong enough - suspends the particles ${ }^{13}$. In our previous studies ${ }^{14-15}$, we dealt with the suspension process for a single particle and a small number of particles; the particles' trajectories and velocities in different stages were measured. Interpretation of the results led to the notion that the suspending force is proportional to the impeller speed to the power 1.4, which is intermediate between viscous and inertial scaling. In addition, it was shown through 
detailed simulation that the pressure field around the particles plays an important role during lift-off.

In the present paper we characterize the initial lift-off of a solid particle through a combination of experiments, modeling, and simulation. We take the seemingly simple situation of a single spherical particle on a flat horizontal bottom of a square container filled with a viscous liquid. To build fundamental understanding of the way forces acting on a particle develop during lift-off, we consider laminar flow. In addition, the direct hydrodynamic environment of small particles (smaller than eddy size) in turbulence is laminar in nature. To have undistorted optical access in order to accurately measure particle trajectories we use a tank with planar walls. We do not expect a fundamental difference in lift-off mechanisms as compared to a cylindrical tank as long as particle sizes are much smaller than the tank size which they are in our study. The vertical motion of the particle very close to the wall has our particular interest. By carefully filming the lift-off process we gain information about the dynamics of the particle and thus the forces acting on the particle. This information we try to match with how the vertical hydrodynamic force on the particles depends on its location on and closely above the bottom wall as derived from detailed numerical simulations.

Our aim thus is to interpret the experimentally observed vertical particle motion in terms of the forces acting on the particle thereby identifying the lift-off mechanism(s). In the first place, highly-resolved visualization experiments are used to obtain detailed information on the initial lift-off of the particle. In the second place, based on experimental observations as well as numerical simulations, a hydrodynamic force model has been devised to accurately reproduce the measured initial lift-off. In the third place, these fundamental results will be a starting point and reference for more complex flow systems involving multiple particles in laminar as well as in turbulent flow. 
This paper is organized in the following manner: in the next section, the experimental flow systems are introduced: particle properties, working fluid, and the way the flow is generated. Thereafter, experimental details and quantitative visualization during the initial lift-off of a particle are presented. The subsequent section gives a brief outline of the particleresolved simulations based on the lattice-Boltzmann method (LBM). The Results and Discussion section is divided in two parts. First, impressions of lift-off experiments are given, such as trajectories for two different particles driven by the flow generated by two types of impeller. At first a simple lubrication force model is used to represent the lift-off trajectories. Second, results of simulations with fixed particles are presented, and the pressure distributions around the particles are inspected. A more detailed force model derived from the simulation results is then used to refine the lift-off model. Finally, the main conclusions and the future plans are listed.

\section{Flow system}

The layout of the flow system is given in Figure 1. It consists of a cubic container with side length $T=220 \mathrm{~mm}$. The tank is filled with a Newtonian liquid with kinematic viscosity $\nu$ (in $\mathrm{m}^{2} / \mathrm{s}$ ) and density $\rho$ (in $\mathrm{kg} / \mathrm{m}^{3}$ ) up to a level $H=T$ where there is a free surface. Figure 1 defines the coordinate system that will be applied throughout this paper. Two types of impeller have been used to generate fluid flow in the tank: a round disk and a Rushton turbine (see Figure 1, right panel). They both have a diameter $D=0.5 T$ and an off-bottom clearance $C=0.25 T$. The Reynolds number of this system is defined as $\operatorname{Re}=N D^{2} / \nu$ with $N$ the angular velocity of the impeller (in rev/s). We used a 6SL3210 frequency converter (Siemens, Germany) to control the impeller speed. When switched on, the impeller linearly ramps up its rotational speed at a rate of $100 \mathrm{rpm} / \mathrm{s}$ until a certain target rotational speed is reached. We control the target speed within $\pm 0.5 \mathrm{rpm}$. 
In the experiment, a spherical particle with diameter $d_{p}$ is placed on the bottom in the centre of the tank $\left(0.0,0.0,0.5 d_{p}\right)$. In order to assure this location of the particle, an initial, low impeller speed $N_{0}$ (to be specified below) was maintained for about $30 \mathrm{~s}$ at the beginning of an experiment. The resulting slow flow moves the particle to the centre of the bottom and keeps it there. After these initial $30 \mathrm{~s}$ we ramp up the impeller speed and monitor lift-off of the particle (more details in the next section).

Two particles have been considered. Both have diameter $d_{p}=10.0 \mathrm{~mm}$; they have different densities. The polymethyl methacrylate (PMMA) particle has $\rho_{p}=1189 \mathrm{~kg} / \mathrm{m}^{3}$, the glass particle has $\rho_{p}=2520 \mathrm{~kg} / \mathrm{m}^{3}$. The presence of a particle gives rise to additional dimensionless numbers characterizing the flow system. For this the density ratio $\rho_{p} / \rho$ and the inertial Shields number $\theta=\rho N^{2} D^{2} /\left(g \Delta \rho d_{p}\right)$ have been chosen, where $g$ is gravitational acceleration (in $\mathrm{m} / \mathrm{s}^{2}$ ) and $\Delta \rho=\rho_{p}-\rho\left(\right.$ in $\left.\mathrm{kg} / \mathrm{m}^{3}\right)$. The inertial Shields number is a measure for the ratio of inertial fluid stress (lifting off the particle) and net gravity (pulling the particle towards the bottom of the tank).

The working fluid is a silicone oil (provided from Shanghai Lubao Company, $\rho=977$ $\mathrm{kg} / \mathrm{m}^{3}$ ); it was chosen for its good stability and transparency. The dynamic viscosity has been measured with a MARS40 rheometer (Haake, Germany). It depends on temperature according to $\mu=3.1790-0.0438 T_{\text {oil }}$ (with viscosity $\mu$ in $\mathrm{Pa} \cdot \mathrm{s}$ and $T_{\text {oil }}$ in ${ }^{\circ} \mathrm{C}$ ) in the range $17^{\circ} \mathrm{C} \leq T_{\text {oil }} \leq 27^{\circ} \mathrm{C}$. In the experiments the liquid temperature was regularly monitored at two locations in the container (see Figure 1). The average temperature of the two locations was controlled at $25.7 \pm 0.1{ }^{\circ} \mathrm{C}$ over the experimental campaign. This implies a dynamic viscosity of $\mu=2.053 \mathrm{~Pa} \cdot \mathrm{s}$ (within a $0.2 \%$ variation) and a kinematic viscosity of $v=2.101 \times 10^{-3} \mathrm{~m}^{2} / \mathrm{s}$. In this study, Reynolds numbers are in the range 8-39 so that the flow conditions in the 
stirred tank are considered laminar. No waves developed on the free surface. Laminar flow was also observed at $\mathrm{Re}=26.04$ in previous work $^{14}$, based on the liquid velocity field. In addition, literature ${ }^{16-18}$ considers that the flow is laminar if the impeller-based Reynolds number is up to 100.

\section{Experimental method and visualization}

The procedure for measuring the lift-off of a particle is as follows: the flow driven by the initial speed $N_{0}$ accurately places the particle in the center of the bottom of the tank. After $30 \mathrm{~s}$ of agitation at speed $N_{0}$, the impeller speed ramps up at a rate of $100 \mathrm{rpm} / \mathrm{s}$ until it reaches a target speed $N$ (this takes a few seconds at most). If the particle is not lifted off in one minute from the moment the target speed is reached, we stop the impeller and repeat the process with an increased target speed, until there is lift-off within one minute. The critical impeller speed for which the particle gets lifted off within one minute we call $N_{L O}$. It is for practical reasons that we limit the lift-off time to one minute. In computational research, such time span is still feasible for particle-resolved simulations of solids suspension processes, longer times become unfeasible. Each experiment is repeated at least two times. The value for $N_{L O}$ has an accuracy - based on reproducibility and the step size with which we increase the target speed - of approximately 3\%. The initial impeller speed enhances the reproducibility of the experiments as it makes sure the particle lifts off from the center of the bottom of the tank. The lift-off process is not sensitive to the precise value of $N_{0}$. Therefore, the initial impeller speed $N_{0}$ typically has been set to $0.5 N_{L O}$. The time measured from the moment we reach the target impeller speed until the particle is lifted off by $1 \mathrm{~mm}$ (which is $0.1 d_{p}$ ) we call $t_{0.1}$.

The vertical lift-off trajectory was filmed with a high-speed camera (Dantec Dynamics A/S, Denmark) with a $180 \mathrm{~mm}$ macro lens (Nikon, Japan). A strong light source with the 
light first passing through a scatter plate (see Figure 2) was used to obtain clear images of the particles. The emphasis in recording the particle motion is on spatial resolution, less so on speed of filming. A rate of 192 frames per second (fps) is sufficient to capture the temporal evolution. This relatively low frame rate allows for filming with a resolution of $2320 \times 1726$ pixel $^{2}$ with $7 \mu \mathrm{m}$ per pixel. A particle diameter thus represents approximately 1430 pixels and we are able to measure particle displacement with a resolution of the order of $10^{-3} d_{p}$. A typical camera image is given in Figure $3 \mathrm{a}$.

The initial lift-off of the particle can be quantified by detecting the edge and centroid of the particle in an image processing procedure. The images were processed in the Matlab environment (version R2017a, Mathworks, USA). The edge detection operator based on Canny ${ }^{19}$ was used for the computation of edge points of the sphere in each camera frame (see Figure $3 b$ ). Thereafter, the centroid of particle was given in the following expression (see

Figure 3c): $\bar{x}=\iint_{(\sigma)} x d \sigma / \iint_{(\sigma)} d \sigma$ and $\bar{z}=\iint_{(\sigma)} z d \sigma / \iint_{(\sigma)} d \sigma$ where $\sigma$ is the field of integration with upper $\left(x_{2}, z_{2}(x)\right)$ and lower $\left(x_{1}, z_{1}(x)\right)$ limits $^{20}$. The uncertainty of the estimate of the centroid of the sphere corresponds to one pixel and thus to $7 \mu \mathrm{m}$.

\section{Numerical Approach}

In order to estimate hydrodynamic forces acting on a spherical particle on and closely above the bottom of the container, numerical simulations of the flow in the mixing tank containing the particle have been performed. The laminar flow in the mixing tank allows us to do direct simulations for which the lattice-Boltzmann method (LBM) has been applied. The specific scheme we use is due to Somers ${ }^{21}$ and Eggels ${ }^{22}$. We have extensive experience with applying this method to stirred tank flow ${ }^{23}$, as well as to solid-liquid suspensions ${ }^{14-15,24}$. The method operates with a uniform, cubic grid the resolution of which is such that the particle diameter is spanned by 12 lattice spacings $\left(d_{p}=12 \Delta\right)$. Earlier work ${ }^{25}$ has shown - 
by grid convergence studies - that such resolution is sufficient for correctly predicting hydrodynamic forces on the particle if particle-based Reynolds numbers are not larger than $\mathrm{O}(30)$. Also in this paper we have verified grid convergence by refining the grid to $d_{p}=20 \Delta$ for a few cases. At the default resolution of $d_{p}=12 \Delta$ the tank volume (a cube with side length $T=220 \mathrm{~mm}$ ) is discretized by a grid of $264^{3}$ cells, and the impeller diameter $D=132 \Delta$

The simulations resolve the flow around the particle, i.e. the computational grid is finer than the size of the particle so that the no-slip condition can be imposed explicitly at its surface and that forces (as well as torques) on the particle are a direct result of the simulations as implemented by the immersed boundary method ${ }^{26-29}$. In this method, set of off-lattice, closely spaced marker points are defined on the particle surface. Interpolated velocity of fluid is forced to the local velocity of solid surface based on a control algorithm in order to achieve the no-slip condition. Integration of forces maintaining no-slip over the particle surface gives the total hydrodynamic forces and torques on the particle. A calibration $\operatorname{step}^{28,30-31}$ at low Reynolds number has been implemented to correct for grid effects.

Also the rotational motion of the impeller was accounted for through the immersed boundary method. The no-slip boundary conditions at the tank walls were applied by means of a halfway bounce-back rule ${ }^{32}$. A halfway specular reflection rule was imposed to achieve the free-slip condition ${ }^{33}$ at the top surface.

Only situations with the particle on the centerline of the tank at fixed vertical locations will be considered in this paper. This is because - as explained in the Results and Discussion section - accelerations in the experiments are such that during the lift-off process inertia forces are 2 orders of magnitude smaller than the lubrication force involved.

To achieve incompressible flow conditions in our slightly compressible LBM simulations, the speed of the fluid $|\mathbf{u}|$ (in lattice units) is required to be much lower than the 
speed of sound which is of order 1 in lattice units ${ }^{28}$. For this reason, the tip speed of the impeller (which is a good measure for the maximum speed occurring in the tank) has been set to 0.1 in lattice units. This then implies that, as an example, it takes 5400 time steps for the case of the glass particle with the Rushton turbine to make one revolution: $N=1 /(5400 \Delta t)$. As a result, the kinematic viscosity is determined as $\nu=0.1139$ (in lattice units) which then gives a Reynolds number ( $\operatorname{Re}=N D^{2} / \nu=28.34$ ) in accordance to the experiments with $N_{L O}=296 \mathrm{rpm}$.

In a simulation, the particle is first placed at a fixed location in a zero-velocity flow field. The impeller motion is started and the flow develops into a quasi-steady state at a prescribed impeller-based Reynolds number. The force on the particle reported in this paper is the force averaged over a full impeller revolution after steady state has been reached.

\section{Results and Discussion}

\section{Analysis of experimental particle trajectories}

First we determined the lift-off impeller speed $\left(N_{L O}\right)$ for the four base-case situations (two impellers, two particles). In addition to experiments at $N_{L O}$, we also report on trajectory visualization experiments at slightly higher impeller speeds. An overview of the in total 12 experimental cases considered in this paper is given in Table 1. The denser glass particle needs a stronger flow to be lifted off so that its $N_{L O}$ is significantly larger than that of the PMMA particle. Clearly the Rushton turbine creates a stronger flow than the disk which leads to lower $N_{L O}$ values for the former. An increased impeller speed gives rise to a much shorter lift-off time $t_{0.1}$.

The position of a particle in the horizontal $x$-direction has been measured during its departure from the bottom wall (see Figure 4). Time $t=0$ corresponds to the moment when the impeller has accelerated to target speed $N$. This definition of $t=0$ will be used in the 
subsequent discussion. As we can see in Figure 4, the particle's location only slightly deviates from $x=0$ at $t=0$. Such deviations are caused by weak perturbations associated with the impeller accelerating from $N_{0}$ to $N$. Before the sphere lifts off the bottom wall, the maximum displacement of the horizontal location of the particle is about $0.31 \mathrm{~mm}$ with the Rushton turbine and $0.26 \mathrm{~mm}$ with the spinning disk. These are relatively small horizontal displacements so that we can state that to a good approximation the particle moves in the $\operatorname{vertical}(z)$ direction only.

Experimental particle trajectories in the form of the distance from the lowest point of the sphere to the container bottom $(h)$ as a function of time $t$ are given in Figure 5. We show two realizations of each experiment and obtain well-reproducible results. At $t=0$, the particle is in contact with the bottom of tank. With the development of the flow field, the vertical hydrodynamic force on the particle overcomes its net gravity, and the particle slowly accelerates away from the bottom wall. For the same particle under the critical lift-off conditions, the flow generated by the spinning disk develops slower than for the Rushton turbine. Taking the critical cases of the PMMA particle as example, more time is needed to lift the particle by $1 \mathrm{~mm}$ for the spinning disk $\left(N_{L O}=93 \mathrm{rpm}\right)$, which means the trajectory of the particle shifts to longer times.

In an elementary description of lift-off and vertical motion of the particle, we assume a hydrodynamic force $F_{h, z}$ acting in the positive $z$-direction that is larger than the net gravity force on the particle: $F_{n e t, z}=F_{h, z}-\pi g d_{p}{ }^{3}\left(\rho_{p}-\rho\right) / 6>0$. The lubrication force on the particle when it moves away from the flat bottom wall with a vertical velocity $u_{z}$ is $F_{l, z}=-\frac{3}{2} \pi \rho \nu d_{p}{ }^{2} u_{z} / h^{6,34}$. In a quasi-steady formulation, $F_{n e t, z}$ balances the lubrication force. Since also $u_{z}=d h / d t$ it can be derived that

$$
\ln \left(h / h_{0}\right)=\frac{2}{3} F_{n e t, z} t /\left(\pi \rho \nu d_{p}^{2}\right)
$$


with $h_{0}$ the spacing between particle and bottom at $t=0$. This initial spacing is an uncertain and unpredictable factor in our analysis as it relates to the roughness of the surfaces of the particle and the container bottom and the precise initial placement of the particle. It is interesting to note that roughness - and therefore a non-zero $h_{0}$ - is an essential feature of the lift-off process. In the hypothetical situation of perfectly smooth surfaces (of particle and bottom plate) lift-off will not be possible. The main assumptions - quasi steady formulation and constant hydrodynamic force - will be revisited below.

Inspired by Eq. 1, the same information as in Figure 5 is plotted in a logarithmic-linear way in Figure 6. In this figure the finite resolution (pixilation) of the imaging system shows as step changes (by $7 \mu \mathrm{m}$ ) for the lower values of $h$. Beyond these steps - starting from approximately $h=0.03 \mathrm{~mm}$ - we see an approximately linear region up to $h=1 \mathrm{~mm}$ for the PMMA particle, in qualitative agreement with Eq. 1, and a strong positive departure from linearity for the glass particle.

From fitting straight lines through the data, examples of which are shown in Figure 6, values for $h_{0}$ and $F_{n e t, z}$ can be derived. The fitting range for the PMMA particle is $0.03 \mathrm{~mm} \leq h \leq 1.0 \mathrm{~mm}$. For the glass particle the data in Figure 6 hardly show linearity and we have limited the fitting range to $0.03 \mathrm{~mm} \leq h \leq 0.2 \mathrm{~mm}$. Estimates for $h_{0}$ and $F_{n e t, z}$ are derived from the intercept and the slope of fitted line respectively. Although the contact point between the sphere and the bottom wall is random in each experiment, the order of magnitude of the fitted initial spacing $h_{0}$ (that we term $h_{0, \mathrm{Eq} .1}$ from now on to distinguish it from other fitting procedures later in this paper) is consistently the same: values for $h_{0, \mathrm{Eq} .1}$ associated to the glass and PMMA sphere are in the range of 2 to $4 \mu \mathrm{m}$. The slope of the fitted line $k$ (in $1 / \mathrm{s}$ ) is positively correlated to the rotational speed of impeller, and the order of magnitude of 
the estimated $F_{n e t, z}$ (given that $k=\frac{2}{3} F_{n e t, z} /\left(\pi \rho \nu d_{p}{ }^{2}\right)$ ) in the 12 experimental cases is of the order of $10^{-4} \mathrm{~N}$.

As mentioned before, we hypothesize that the estimated $h_{0, \mathrm{Eq} .1}$ values are related to surface roughness. This is supported by experimental data on the surface roughness of three samples as measured by means of atomic force microscope (AFM) in a $70 \times 70 \mu \mathrm{m}^{2}$ area (DMFASTSCAN2-SYS, Bruker, Germany). As we can see in Figure 7, the surfaces of the glass and PMMA particle are rougher than the sheet of glass used as the bottom wall. The absolute values for arithmetic average roughness $R_{a}{ }^{35}$ are $1.58 \mu \mathrm{m} 1.27 \mu \mathrm{m}$, and $0.21 \mu \mathrm{m}$ for the PMMA particle, the glass particle, and the sheet of glass respectively. These are roughness values comparable to $h_{0, \mathrm{Eq} .1}$ as obtained from the fitting procedure and thus supportive of our hypothesis.

The departures from linearity as witnessed in Figure 6 - most strongly for the glass particle - show that the analysis based on a force balance resulting in the trajectory equation (Eq. 1) is not capturing the essential physics very well. In the next section we will be analysing the lift-off process in more detail by involving results obtained from particleresolved numerical simulations.

\section{Analysis of lift-off process}

In order to justify a quasi-steady approach, the acceleration term in the particle's vertical equation of motion is compared to the forces on the particle. As representative for the forces on the particle, the lubrication force is considered. It has been estimated based on the vertical velocity $u_{z}=d h / d t$ where for calculating the time derivative we use a $4^{\text {th }}$ order central difference formula that is less sensitive to noise in the position data compared to $2^{\text {nd }}$ order central differences. Further, the acceleration $a_{z}(t)$ of the particle is estimated by taking 
the first derivative of the measured $u_{z}(t)$ versus $t$ curves (with $2^{\text {nd }}$ order central differences). For the three sample experiments of the glass particle (that has higher inertia than the PMMA particle) with the Rushton turbine, the vertical velocity as well as the inertial force as a function of the distance of particle are given in Figure 8. Taking the critical case of the glass particle with $N_{L O}=296 \mathrm{rpm}$ as example, a vertical velocity of the order of $u_{z}=0.4 \mathrm{~mm} / \mathrm{s}$ at a distance $h=0.5 \mathrm{~mm}$ implies a lubrication force of $8 \times 10^{-4} \mathrm{~N}$. This is more than two orders of magnitude larger than the inertial forces shown in Figure 8 (right panel). Also, the estimated $F_{n e t, z}$ in Eq. 1 from the fits in Figure 6 are larger (by two orders of magnitude) than the inertia force $m a_{z}$. We acknowledge the limited accuracy of the experimental particle acceleration data but note that we use them only in an order-of-magnitude sense.

The assumption of a constant hydrodynamic force that led to Eq. 1 has been tested through numerical simulations according to the methodology briefly described in Section 4 . In the simulations we place the particle at fixed locations - in line with the quasi-steady assumption - and "measure" the vertical force on the particle due to the flow generated by the revolving impeller. Particles are placed on the tank centreline with four distances $(h=0$, $0.36 \Delta, 0.72 \Delta$, and $1.2 \Delta$ ), i.e. a height range of $0 \leq h \leq 0.1 d_{p}$. According to the experiences in our previous numerical works ${ }^{14-15}$, a Shields number $\theta_{\text {sim }}$ in simulations which is $7 \%$ higher than in experiments $\theta_{\exp }$ assured that the hydrodynamic force at $h=0$ overcomes net gravity of the particle; $7 \%$ in Shields number corresponds to $3.4 \%$ in impeller speed. For a typical situation the flow field underneath the impeller and around the particle is shown in Figure 9. During the initial lift-off process, a region with higher flow velocity above the particle compared with a flow below the particle results in the negative $\partial P / \partial z$ around the particle. Consequently, the pressure distribution contributes to the initial lift-off. The 
bottom panels of Figure 9 suggest that the effect of pressure gradient on the lift-off is more significant for these cases that have lower Reynolds number.

To understand (non) linearity of $h$ versus $t$ in Figure 6, the vertical hydrodynamic forces at the four distances considered in the simulation are shown in Figure 10. For the case with $\operatorname{Re}=28.34$ (corresponding to $N=296 \mathrm{rpm}$ in the experiment) a finer grid $\left(d_{p}=20 \Delta\right)$ was used to evaluate the influence of grid refinement on the results. As we can see in Figure 10 , refinement of grid has hardly any effect on $F_{h, z}$; the difference between the hydrodynamic force obtained on the fine and standard grid $\left(d_{p}=12 \Delta\right)$ at $h=0$ is less than $0.5 \%$. Therefore, the numerical simulations with $d_{p}=12 \Delta$ resolve the hydrodynamic force on the particle adequately.

In Figure 10 we observe an approximately linear increase of the hydrodynamic forces with the height, which provides inspiration for a more refined lift-off model. In this model, $F_{n e t, z}=F_{0}+\alpha h$, where $\alpha$ is the slope of lines given in Figure 10, and $F_{0}$ is the difference between the hydrodynamic force $F_{h, z 0}$ at $h=0$ and the net gravity force on the particle. The steep changes of net force with the distance from the bottom wall relate to the evolving pressure distribution around the particle in Figure 9 - once the particle has lifted off the bottom.

An analysis based on the linear force model leads to the following trajectory equation:

$$
\ln \left(\frac{h}{h_{0}} \frac{\left(F_{0}+\alpha h_{0}\right)}{\left(F_{0}+\alpha h\right)}\right)=\frac{2}{3} F_{0} t /\left(\pi \rho \nu d_{p}{ }^{2}\right)
$$

A user-defined function for nonlinear fitting provided by toolbox "cftool",36 in the Matlab environment (version R2017a, Mathworks, USA) was used to estimate the three coefficients $\left(h_{0}, F_{0}\right.$ and $\left.\alpha\right)$ in Eq. 2 based on the experimental $h$ versus $t$ data. In the fitting process, the initial value of $h_{0}$ is equal to $h_{0, \mathrm{Eq} .1}$ and the initial values for $F_{0}$ and $\alpha$ are based 
on the relationship between the hydrodynamic force and the distance as observed in the simulations and as shown in Figure 10. As shown in Figure 11, Eq. 2 is very well able to represent the experimental height versus time data. Clearly the upward curvature in the linlog plots as observed in the visualization experiments is the result of an increase of the vertical hydrodynamic force with height. Figure 11 as well as Table 2 provide data for the coefficients $h_{0}, F_{0}$ and $\alpha$ contained in Eq. 2 and shows how they relate to the various experimental conditions. The experimental data and therefore the fitting parameters come with uncertainties. Uncertainties in the fitting parameters have been estimated through the prediction bounds procedure for the fitted curve, which is provided by a Matlab function "predint" ${ }^{37}$. In an absolute sense uncertainties in $\alpha$ are in the range $0.02-0.11 \mathrm{~N} / \mathrm{m}$, uncertainties in $F_{0}$ are of the order of $5 \cdot 10^{-5} \mathrm{~N}$, and in $h_{0}$ they are approximately $4 \mu \mathrm{m}$. Given that $\alpha$ associated with the PMMA particle experiments is one order of magnitude smaller than $\alpha$ for glass particle experiments, the relative uncertainty in $\alpha$ for PMMA is much higher than for glass (at most $30 \%$ and $10 \%$ for PMMA and glass respectively). In what follows the data in Table 2 will be interpreted quantitatively in light of the force estimates of the numerical simulations as well as data on the surface roughness of the particles.

The values for $h_{0}$ associated to the glass and PMMA sphere are consistently in the range of $2 \mu \mathrm{m}$ to $5 \mu \mathrm{m}$. The asperities on the surface of the glass particle are given in the middle panel of Figure 7; the bottom middle panel of Figure 7 shows that the roughness structures on the surface cover a range of approximately $5 \mu \mathrm{m}$ so that it is reasonable to associate the $h_{0} \approx 3 \mu \mathrm{m}$ for glass with its surface roughness. Similar results have also presented in AFM measurement of PMMA particle; that is, $h_{0} \approx 4 \mu \mathrm{m}$ for PMMA is reasonable. 
When it comes to $\alpha$, in the first place we see it does not strongly depend on the impeller speed for each of the four impeller/particle combinations; for Rushton turbinePMMA, disk-PMMA, Rushton turbine-glass, and disk-glass fitted $\alpha$ values of approximately $0.14,0.15,1.4$ and $1.3 \mathrm{~N} / \mathrm{m}$ are found respectively. The three impeller speeds per impeller/particle combination are relatively close to one another so that the flow in the tank and therefore $\alpha$ is not expected to show much variation. The estimates for $\alpha$ based on the fits in Figure 11 are in line with what was observed in the numerical simulations. For the Rushton-glass combination we have the most extensive set of data for the vertical hydrodynamic force. At impeller speeds 296, 316, and $336 \mathrm{rpm}$ values of $\alpha$ of 1.61, 1.70, and 1.79 have been derived from the simulations. These values are about 1.2 times the values from fitting the height versus time curve; their variation with impeller speed is small, as expected. A similar picture emerges for the data of glass particle with the spinning disk. It is, however, notable that simulated values of $\alpha$ for the PMMA particle are approximately twice their fitted results. One should realize the large relative uncertainties in the fitted values of $\alpha$ in the experiments involving a PMMA particle (30\%) as well as a slight but systematic mismatch between simulation and experiment that led us to simulate with a $7 \%$ higher Shields number $(\theta)$ as compared to the experiments ${ }^{14}$.

For interpretation of $F_{0}$, we include the total hydrodynamic force at $h=0$ from fitting and from the simulations: $F_{h, z 0}=F_{0}+\pi g\left(\rho_{p}-\rho\right) d_{p}^{3} / 6$ in Table 2. The fitted $F_{h, z 0}$ agrees with simulated results within $15 \%$. We plot the fitted force $F_{h, z 0}$ against the impeller speed in Figure 12 on double logarithmic scales. Given the limited ranges of impeller speeds per impeller-particle combination we cannot be that conclusive with respect the way the force depends on the impeller speed. Clearly Figure 12 indicates that the force increases with impeller speed tentatively showing a dependency $N^{\beta}$ with $\beta$ between 0.6 and 2 . 


\section{Summary \& conclusions}

Suspending solid particles in a liquid is an important way of enhancing solid-liquid mass transfer, for instance in catalytic chemical reactors with solid particles impregnated by a catalyst. In reactors, stirring is a common way of creating a fluid flow that is strong enough to lift particles off the bottom of the reactor. In modeling the initial stages of the solids suspension process, lubrication force models are a useful concept. This paper explores through highly-resolved visualization experiments - the accuracy and usefulness of lubrication force models for describing lift-off of spherical solid particles in the vicinity of a solid surface due to a flow generated by a revolving impeller. Since lubrication forces diverge for zero spacing between the reactor bottom surface and the particle, surface roughness and thus an initial finite spacing is an important parameter in the lift-off process.

From the visualization experiments, time series of the spacing between the particle and the bottom surface (symbol $h$ ) were derived. The very initial stages of lift-off show the expected linear behavior between time and $\ln \left(h / h_{0}\right)$ with $h_{0}$ the initial spacing, confirming that lubrication is a dominant phenomenon at lift-off. Fitting the time series with a straight line then provides quantitative information of the vertical hydrodynamic force on the particle as well as on the initial spacing. The latter could be related to the surface roughness of the particle, which was measured independently.

Dependent on the particle density, upward deviations from linearity in the time versus graphs were observed (e.g. the particle with higher inertia), starting at spacing as small as about 0.02 times the particle diameter. This indicates that the vertical hydrodynamic force on the particle increased strongly over a small vertical displacement of the particle. The extent of this effect was investigated by means of numerical simulations fully resolving the flow generated through agitation as well as the flow around the particle. The simulations revealed that the hydrodynamic force indeed increased with the spacing between particle and bottom 
wall, approximately in a linear fashion. It was shown that the pressure distribution around the particle plays an important role in this effect. Finally, the relationship between force and spacing $(h)$ as derived from the simulations allowed us to quantitatively predict the experimental lift-off observations by solving the ordinary differential equation describing the vertical component of the equation of motion of the particle and involving a linear increase of hydrodynamic force with $h$.

The results and interpretations presented in this paper have significance for designing and assessing simulations of solids suspension processes. We conclude that small-scale variations of forces on particles play an important role in the suspension process. According to the "base-case" results obtained in this paper, future research will focus on more complex systems, including different types of impellers creating different types of flow, most notably systems involving multiple particles where interaction between particles will have impact on the way the solids get suspended in the liquid.

\section{Acknowledgement}

The financial support from the National Key R\&D Program of China (2016YFB0302801), National Natural Science Foundation of China (No. 21676007), and the Fundamental Research Funds for the Central Universities (XK1802-1) are gratefully acknowledged.

\section{References}

1. Leal LG. Particle motions in a viscous fluid. Annu Rev Fluid Mech. 1980;12:435-476.

2. Feuillebois F. Some theoretical results for the motion of solid spherical particles in a viscous fluid. Multiphas Sci Technol. 1989;4:583-789.

3. Michaelides EE. Review-The transient equation of motion for particles, bubbles, and droplets. J Fluids Eng. 1997;119:233-247.

4. Goldman AJ, Cox RG, Brenner H. Slow viscous motion of a sphere parallel to a plane wall-I Motion through a quiescent fluid. Chem Eng Sci. 1967;22:637-651.

5. Brenner H. The slow motion of a sphere through a viscous fluid towards a plane surface. Chem Eng Sci. 1961;16:242-251. 
6. Cox RG, Brenner H. The slow motion of a sphere through a viscous fluid towards a plane surface-II Small gap widths, including inertial effects. Chem Eng Sci. 1967;22:17531777.

7. Rabinovich E, Kalman H. Incipient motion of individual particles in horizontal particlefluid systems: A. Experimental analysis. Powder Technol. 2009;192:318-325.

8. Rabinovich E, Kalman H. Incipient motion of individual particles in horizontal particlefluid systems: B. Theoretical analysis. Powder Technol. 2009;192:326-338.

9. Soepyan FB, Cremaschi S, Sarica C, Subramani HJ, Kouba GE. Solids transport models comparison and fine-tuning for horizontal, low concentration flow in single-phase carrier fluid. AIChE J. 2014;60:76-122.

10. Henry C, Minier JP. Progress in particle resuspension from rough surfaces by turbulent flows. Prog Energ Combust. 2014;45:1-53.

11. Nasr B, Ahmadi G, Ferro AR, Dhaniyala S. Overview of mechanistic particle resuspension models: comparison with compilation of experimental data. J Adhes Sci Technol. 2019;33:2631-2660.

12. Shnapp R, Liberzon A. A comparative study and a mechanistic picture of resuspension of large particles from rough and smooth surfaces in vortex-like fluid flows. Chem Eng Sci. 2015;131:129-137.

13. Zamzam Z, Yoshikawa S, Ookawara S, Kato Y. A new model for estimation of justsuspension speed based on lift force for solid-liquid suspension in a stirred tank. J Chem Eng Jpn. 2016;49:737-746.

14. Mo J, Gao Z, Bao Y, Li Z, Derksen JJ. Suspending a solid sphere in laminar inertial liquid flow-experiments and simulations. AIChE J. 2015;61:1455-1469.

15. Wang C, Zhang L, Li Z, Gao Z, Derksen JJ. Multi-particle suspension in a laminar flow agitated by a Rushton turbine. Chem Eng Res Des. 2018;132:831-842.

16. Lamberto DJ, Alvarez MM, Muzzio FJ. Experimental and computational investigation of the laminar flow structure in a stirred tank. Chem Eng Sci. 1999;54:919-942.

17. Zalc JM, Alvarez MM, Muzzio FJ. Extensive validation of computed laminar flow in a stirred tank with three Rushton turbines. AIChE J. 2001;47:2144-2154.

18. Takahashi K, Sugo Y, Takahata Y, Sekine H, Nakamura M. Laminar mixing in stirred tank agitated by an impeller inclined. Int J Chem Eng. 2012;2012:1-10.

19. Canny J. A computational approach to edge detection. IEEE T Pattern Anal. 1986;8:679698. 
20. Liang W, Wang D, Cai Z, Li Z, Huang X, Gao Z, Derksen JJ, Komrakova, AE. Deformation and breakup of single drop in laminar and transitional jet flows. Chem Eng J. 2019; doi: https://doi.org/10.1016/j.cej.2019.05.173.

21. Somers JA. Direct simulation of fluid flow with cellular automata and the latticeBoltzmann equation. Appl Sci Res. 1993;51:127-133.

22. Eggels JGM, Somers JA. Numerical simulation of free convective flow using the latticeBoltzmann scheme. Int J Heat Fluid Fl. 1995;16:357-364.

23. Zhang Y, Gao Z, Li Z, Derksen JJ. Transitional flow in a Rushton turbine stirred tank. AIChE J. 2017;63:3610-3623.

24. Li G, Li Z, Gao Z, Wang J, Bao Y, Derksen JJ. Particle image velocimetry experiments and direct numerical simulations of solids suspension in transitional stirred tank flow. Chem Eng Sci. 2018;191:288-299.

25. Derksen JJ. Highly resolved simulations of solids suspension in a small mixing tank. AIChE J. 2012;58:3266-3278.

26. Goldstein D, Handler R, Sirovich L. Modeling a no-slip flow boundary with an external force field. J Comput Phys. 1993;105:354-366.

27. Derksen, JJ, Van den Akker HEA. Large eddy simulations on the flow driven by a Rushton turbine. AIChE J. 1999;45:209-221.

28. Ten Cate A, Nieuwstad CH, Derksen JJ, Van den Akker HEA. Particle imaging velocimetry experiments and lattice-Boltzmann simulations on a single sphere settling under gravity. Phys Fluids. 2002;14:4012-4025.

29. Feng ZG, Michaelides EE. Robust treatment of no-slip boundary condition and velocity updating for the lattice-Boltzmann simulation of particulate flows. Comput Fluids. 2009;38:370-381.

30. Sangani AS, Acrivos A. Slow flow through a periodic array of spheres. Int J Multiphas Flow. 1982;8:343-360.

31. Ladd AJC. Numerical simulations of particulate suspensions via a discretized Boltzmann equation. Part 1. Theoretical foundation. J Fluid Mech. 1994;271:285-309.

32. Ziegler DP. Boundary conditions for lattice Boltzmann simulations. J Stat Phys. 1993;71:1171-1177.

33. Succi S. The Lattice Boltzmann Equation for Fluid Dynamics and Beyond. Oxford: Clarendon Press, 2001.

34. Kim S, Karrila SJ. Microhydrodynamics: Principles and Selected Applications. Boston, MA: Butterworth-Heinemann, 1991. 
35. Whitehouse D. Surfaces and Their Measurement. London: Hermes Penton Ltd, 2002.

36. MathWorks Inc. Fit curve or surface to data. Available at: https://uk.mathworks.com/help/curvefit/fit.html. Accessed on 25 June 2019.

37. MathWorks Inc. Prediction intervals for cfit or sfit object. Available at: https://uk.mathworks.com/help/curvefit/cfit.predint.html. Accessed on 25 July 2019. 


\section{List of Figure and Table Captions}

Figure 1. Flow geometry: square-based mixing tank with liquid level equal to the tank's side length $H=T=220 \mathrm{~mm}$. The flow is driven by a disk or by a Rushton turbine (right). The liquid temperature is monitored at two points in the flow. The origin of the coordinate system is at the centre on the tank bottom.

Figure 2. Arrangement for visualization: a camera was used to record the initial lift-off of a sphere from the side; strong light entered into the tank across a scatter plate.

Figure 3. Image of the particle and image analysis for determining the particle position: (a) raw camera frame; (b) the camera frame after Canny detection; (c) image with fitted edge (green circle) and calculated centroid (red star). The left and right boundaries $\left(x_{1}\right.$ and $\left.x_{2}\right)$, vertical position $\left(z_{1}(x)\right.$ and $\left.z_{2}(x)\right)$, and field of integration $\sigma$ as indicated in the panel $\mathrm{b}$.

Figure 4. Location of a particle in horizontal $x$-direction as a function of time. In the left panel the flow is generated by the Rushton turbine (the "RT" is abbreviation of Rushton turbine). In the right panel the flow is generated by the spinning disk. The end of data corresponds to the moment when the distance from the lowest point of the sphere to the container bottom is $1 \mathrm{~mm}$.

Figure 5. The spacing between the lowest point of a sphere and the bottom wall as a function of time on a linear scale. The ' $\mathrm{R}$ ' in the panel means another realization of the experiment.

Figure 6. The spacing between the lowest point of a sphere and the bottom wall as a function of time with a logarithmic scale on the vertical axis. The data between two pink dashed lines 
are used to perform a linear fit by Eq. 1. For the cases of a glass particle, the fitted range of spacing is $0.03 \mathrm{~mm}$ to $0.2 \mathrm{~mm}$; for PMMA, it is $0.03 \mathrm{~mm}$ to $1.0 \mathrm{~mm}$. The black dashed lines are fitted lines. The ' $k$ ' in the panel is the slope of the fitted line. The pink points are the intercept of the fitted lines, from which an estimated initial spacing $h_{0, \mathrm{Eq} .1}$ can be obtained.

Figure 7. Surface characteristics measured by AFM in a $70 \times 70 \mu \mathrm{m}^{2}$ area. Top row: illuminated AFM images; bottom row: height distribution. Left column: the $10 \mathrm{~mm}$ PMMA particle; middle column: the $10 \mathrm{~mm}$ glass particle; right column: glass sheet as used as bottom wall.

Figure 8. Vertical velocity (left) and total vertical force (right) of a glass sphere with Rushton turbine as a function of $h$. The velocity $u_{z}(t)$ is obtained by taking the derivative of $h(t)$ with a $4^{\text {th }}$ order central difference formula. Then $a_{z}(t)$ is calculated by taking the derivative of $u_{z}(t)$ with a $2^{\text {nd }}$ order central difference scheme.

Figure 9. Dimensionless velocity vector and pressure difference around a particle with the Rushton turbine. Top row: $\operatorname{Re}=28.34$ and $\theta_{\text {sim }}=2.03$; bottom row: $\operatorname{Re}=8.14$ and $\theta_{\text {sim }}=1.22$. The spacing between the lowest position of the sphere and the bottom wall in the left, the middle, and the right columns are $0.36 \Delta, 0.72 \Delta$, and $1.2 \Delta$ respectively. Two tip speeds of the impeller $v_{\text {tip } 1}=1.70 \mathrm{~m} / \mathrm{s}$ and $v_{\text {tip } 2}=0.49 \mathrm{~m} / \mathrm{s}$ as indicated.

Figure 10. Symbols: simulated hydrodynamic force on a sphere as a function of height at the specified Reynolds number. Lines: linear fit of the symbols with intercept $F_{h, z 0}$ and slope $\alpha$. 
The resolution of each simulation is indicated by the number of lattice spacings $\Delta$ per particle diameter.

Figure 11. The spacing of the particle as a function of time with a logarithmic scale on the vertical axis, where $\alpha, F_{0}$, and $h_{0}$ are fitted by Eq. 2 . The data (from $h=0.03 \mathrm{~mm}$ to 1.0 $\mathrm{mm}$ ) between two pink dashed lines are used to perform the nonlinear fitting. The pink point represents $h_{0}$.

Figure 12. Vertical hydrodynamic force $F_{h, z 0}$ as a function of impeller speed $N$ as obtained through fitting height versus time. Black symbols: RT-glass; red symbols: Disk-glass; blue: RT-PMMA; green: Disk-PMMA.

Table 1. Experimental parameters and results for the lift-off impeller speed $N_{L O}$ and the impeller speed which is larger than $N_{L O}$.

* After the target impeller speed $N$ is achieved, $t_{0.1}$ is defined as the moment that the particle is lifted off by $1 \mathrm{~mm}$ (which is $0.1 d_{p}$ ).

Table 2. Parameters and results used in experiments, fitting process, and simulations.

* The finer resolution of 20 grid spacings over a sphere diameter is used in the simulations of the fixed glass particle with $\mathrm{Re}=28.34$

The value for $h_{0, \mathrm{Eq} .1}$ is estimated by Eq. 1

The value for $h_{0}$ is nonlinearly fitted by Eq. 2

$\theta_{\text {sim }}=1.07 \theta_{\text {exp }}$ 


\section{Figure 1}

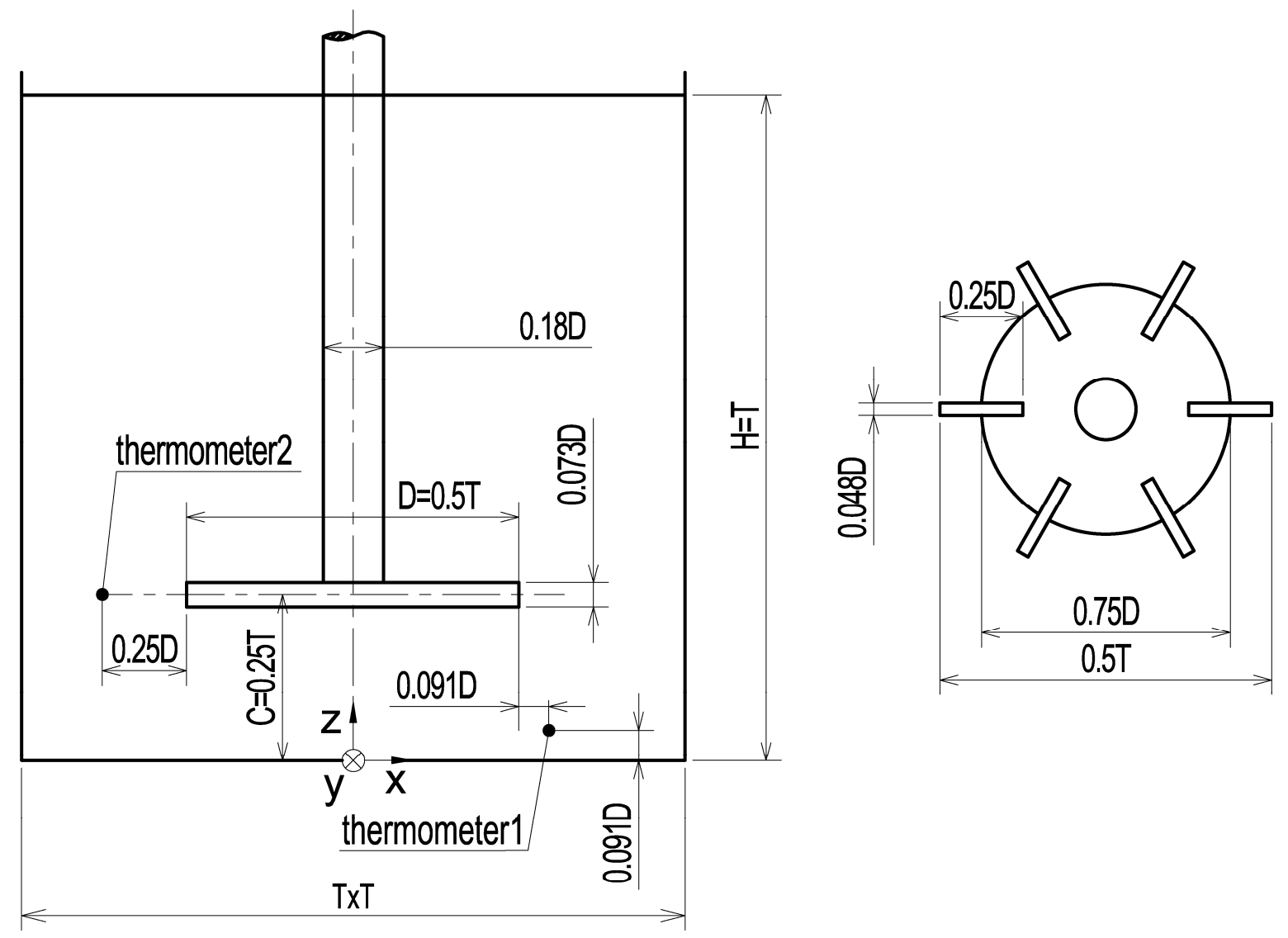

Figure 1. Flow geometry: square-based mixing tank with liquid level equal to the tank's side length $H=T=220 \mathrm{~mm}$. The flow is driven by a disk or by a Rushton turbine (right). The liquid temperature is monitored at two points in the flow. The origin of the coordinate system is at the centre on the tank bottom. 


\section{Figure 2}

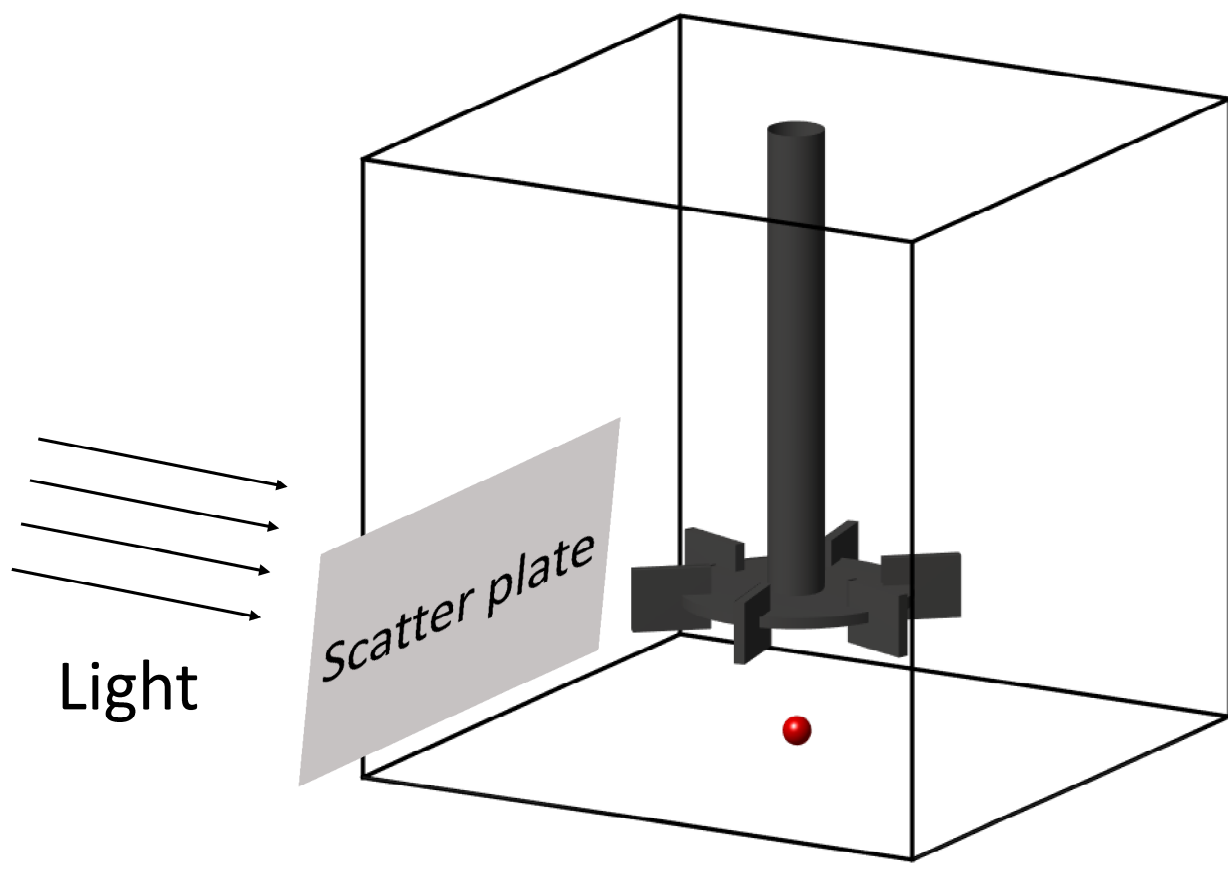

Camera

Figure 2. Arrangement for visualization: a camera was used to record the initial lift-off of a sphere from the side; strong light entered into the tank across a scatter plate. 


\section{Figure 3}
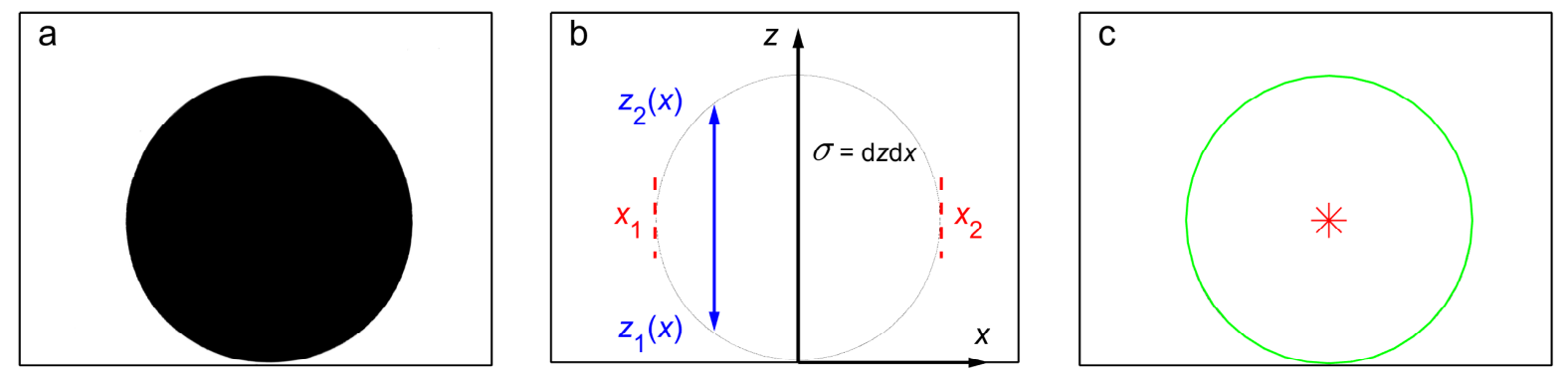

Figure 3. Image of the particle and image analysis for determining the particle position: (a) raw camera frame; (b) the camera frame after Canny detection; (c) image with fitted edge (green circle) and calculated centroid (red star). The left and right boundaries $\left(x_{1}\right.$ and $\left.x_{2}\right)$, vertical position $\left(z_{1}(x)\right.$ and $\left.z_{2}(x)\right)$, and field of integration $\sigma$ as indicated in the panel b. 


\section{Figure 4}
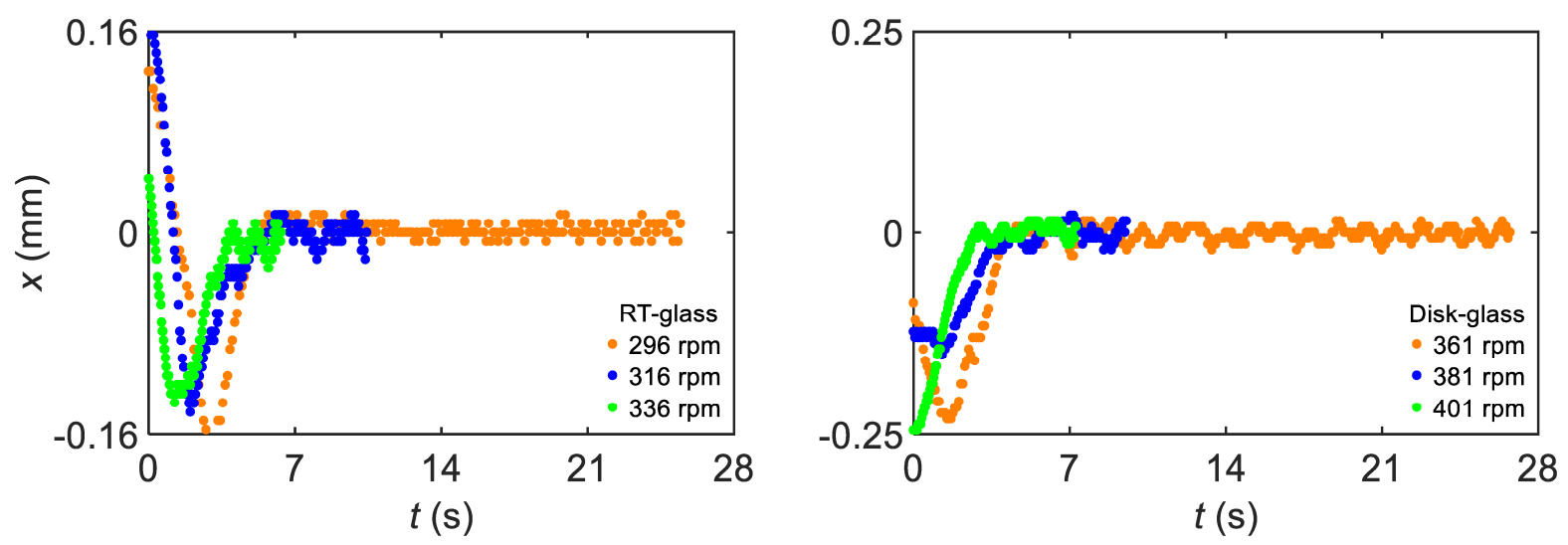

Figure 4. Location of a particle in horizontal $x$-direction as a function of time. In the left panel the flow is generated by the Rushton turbine (the "RT" is abbreviation of Rushton turbine). In the right panel the flow is generated by the spinning disk. The end of data corresponds to the moment when the distance from the lowest point of the sphere to the container bottom is $1 \mathrm{~mm}$. 


\section{Figure 5}
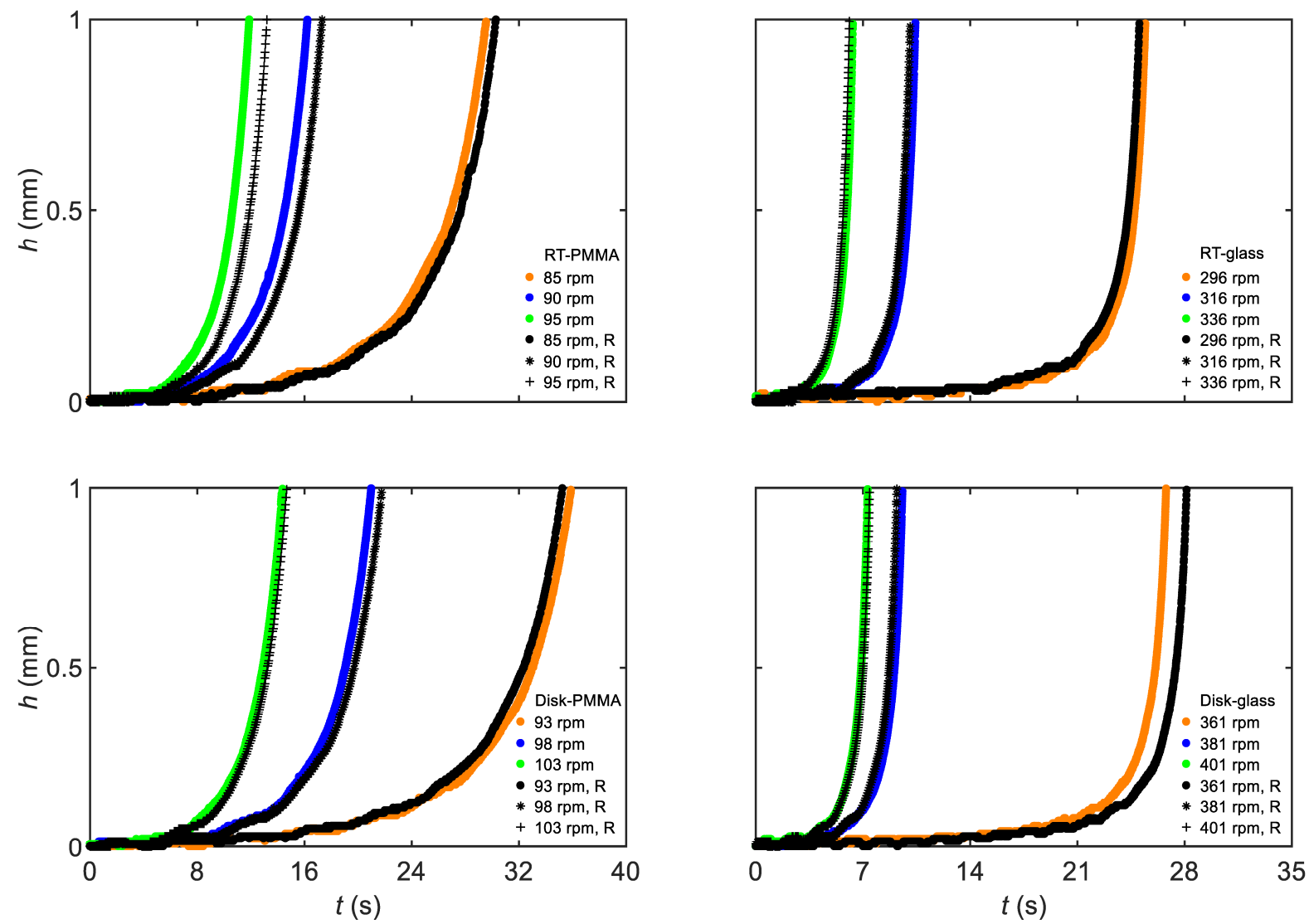

Figure 5. The spacing between the lowest point of a sphere and the bottom wall as a function of time on a linear scale. The ' $\mathrm{R}$ ' in the panel means another realization of the experiment. 


\section{Figure 6}
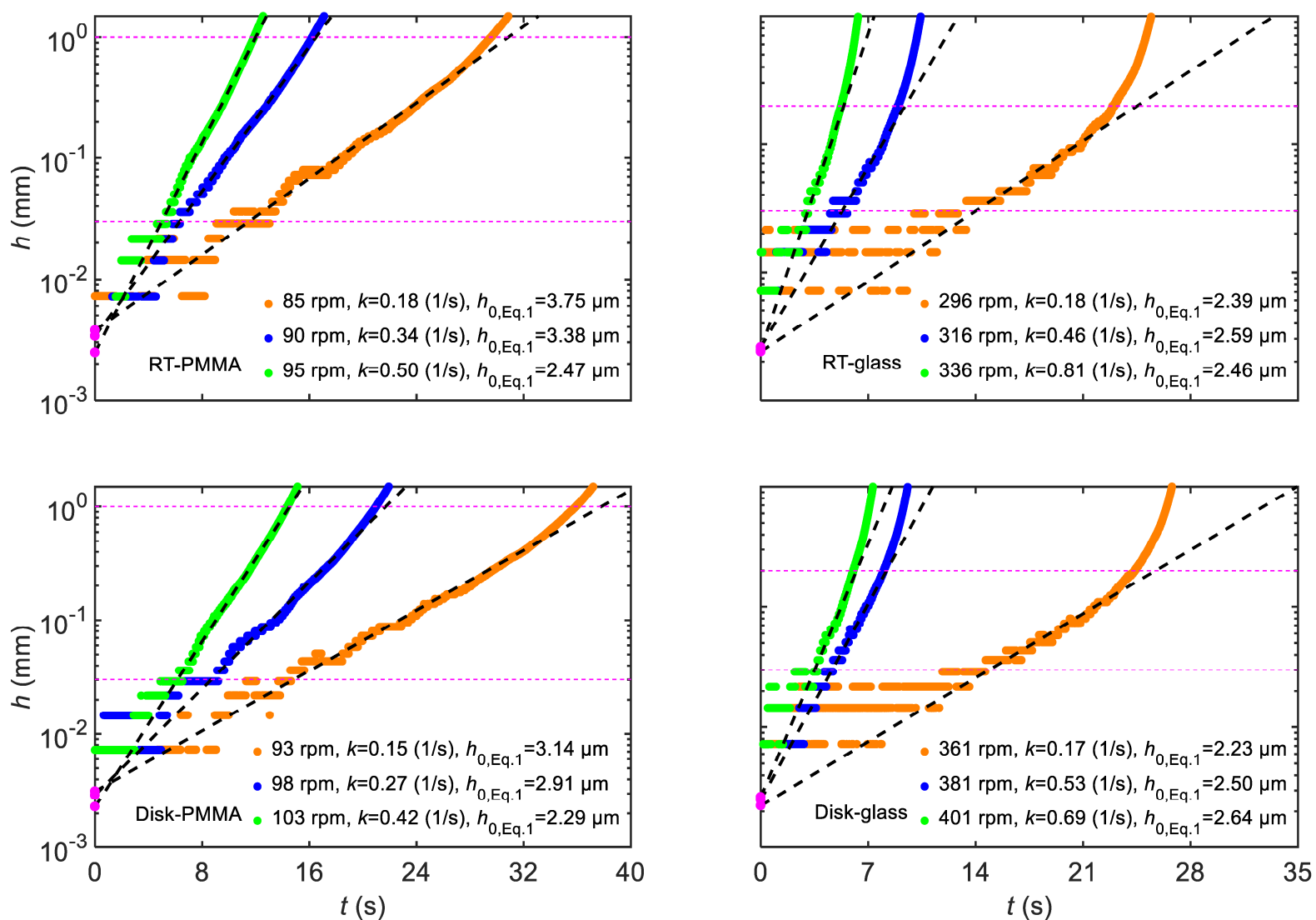

Figure 6. The spacing between the lowest point of a sphere and the bottom wall as a function of time with a logarithmic scale on the vertical axis. The data between two pink dashed lines are used to perform a linear fit by Eq. 1. For the cases of a glass particle, the fitted range of spacing is $0.03 \mathrm{~mm}$ to $0.2 \mathrm{~mm}$; for PMMA, it is $0.03 \mathrm{~mm}$ to $1.0 \mathrm{~mm}$. The black dashed lines are fitted lines. The ' $k$ ' in the panel is the slope of the fitted line. The pink points are the intercept of the fitted lines, from which an estimated initial spacing $h_{0, \mathrm{Eq} .1}$ can be obtained. 


\section{Figure 7}
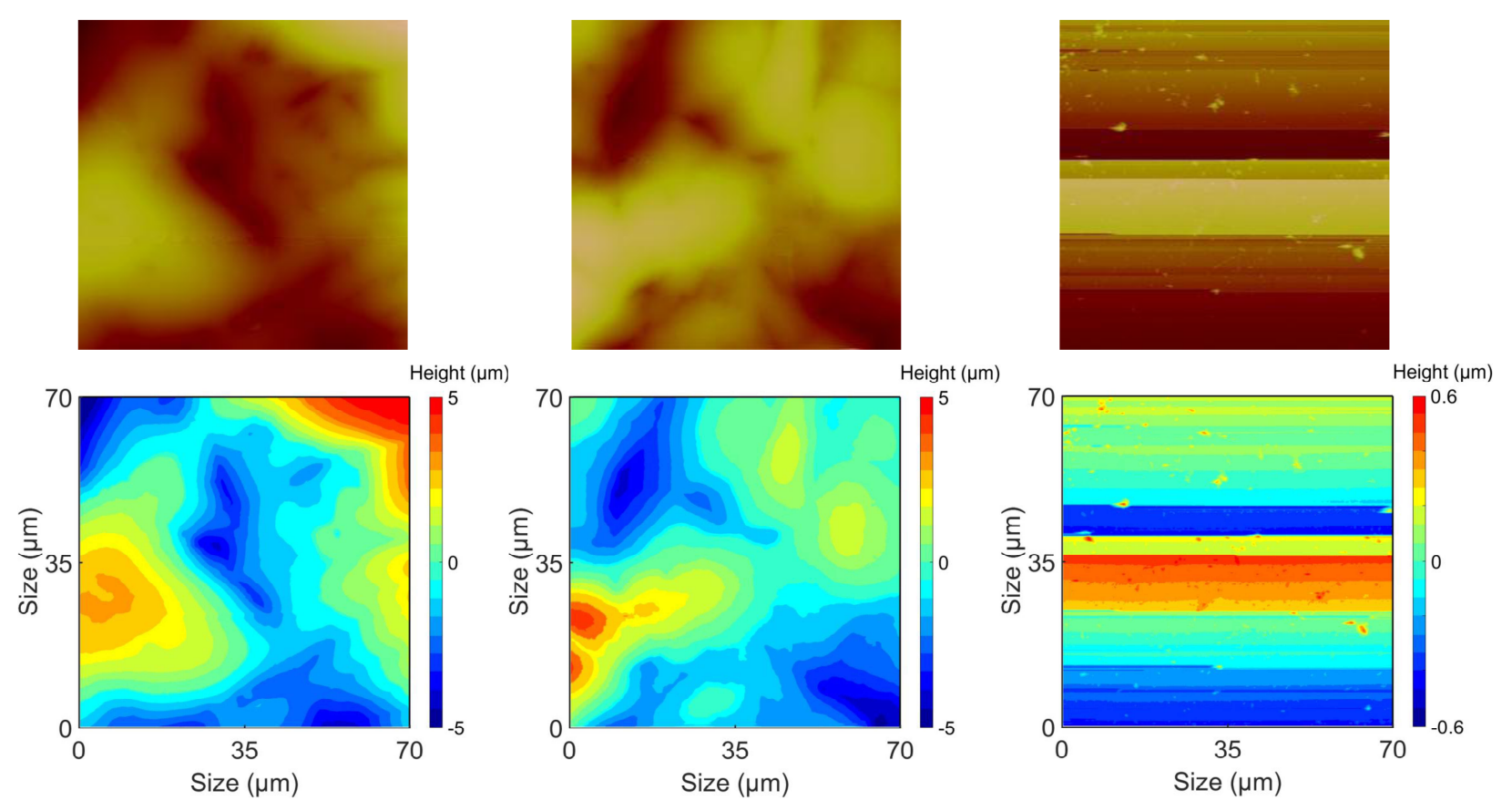

Figure 7. Surface characteristics measured by AFM in a $70 \times 70 \mu \mathrm{m}^{2}$ area. Top row: illuminated AFM images; bottom row: height distribution. Left column: the $10 \mathrm{~mm}$ PMMA particle; middle column: the $10 \mathrm{~mm}$ glass particle; right column: glass sheet as used as bottom wall. 


\section{Figure 8}
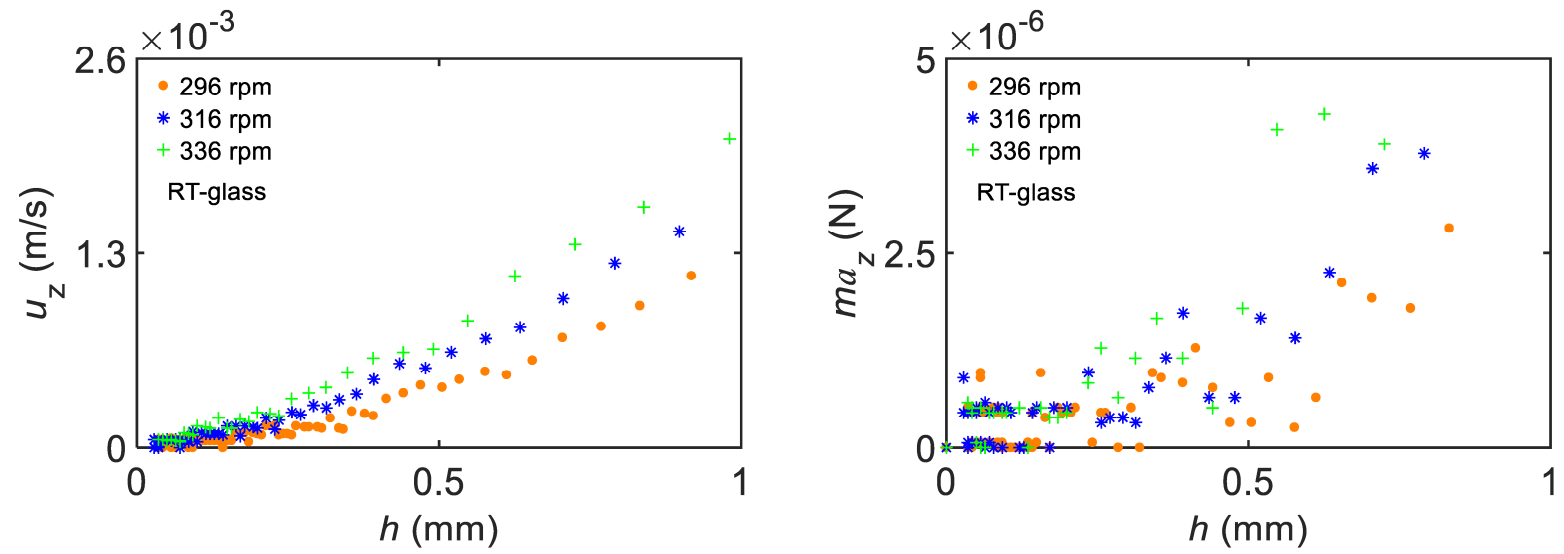

Figure 8. Vertical velocity (left) and total vertical force (right) of a glass sphere with Rushton turbine as a function of $h$. The velocity $u_{z}(t)$ is obtained by taking the derivative of $h(t)$ with a $4^{\text {th }}$ order central difference formula. Then $a_{z}(t)$ is calculated by taking the derivative of $u_{z}(t)$ with a $2^{\text {nd }}$ order central difference scheme. 


\section{Figure 9}
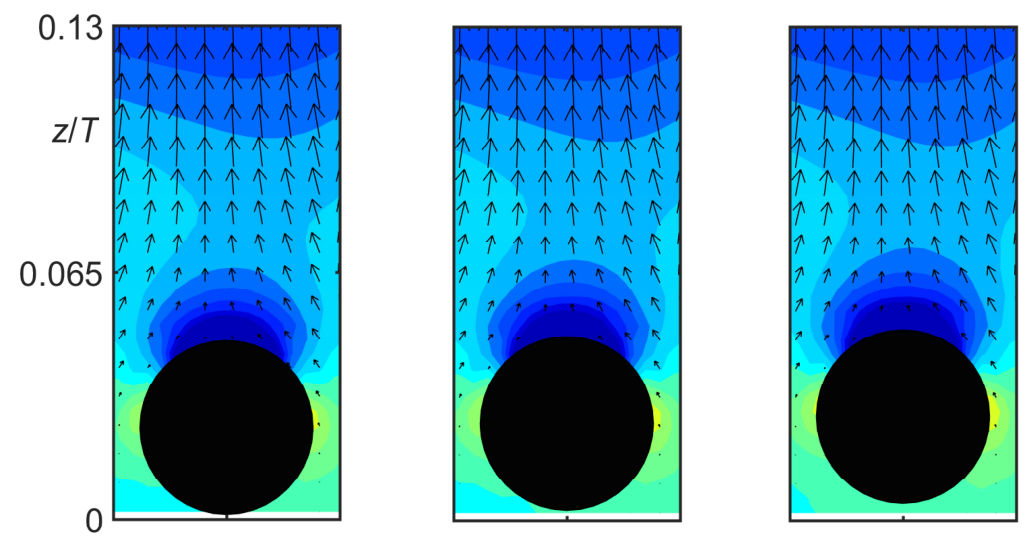

$\uparrow 0.1 v_{\text {tip } 1}$
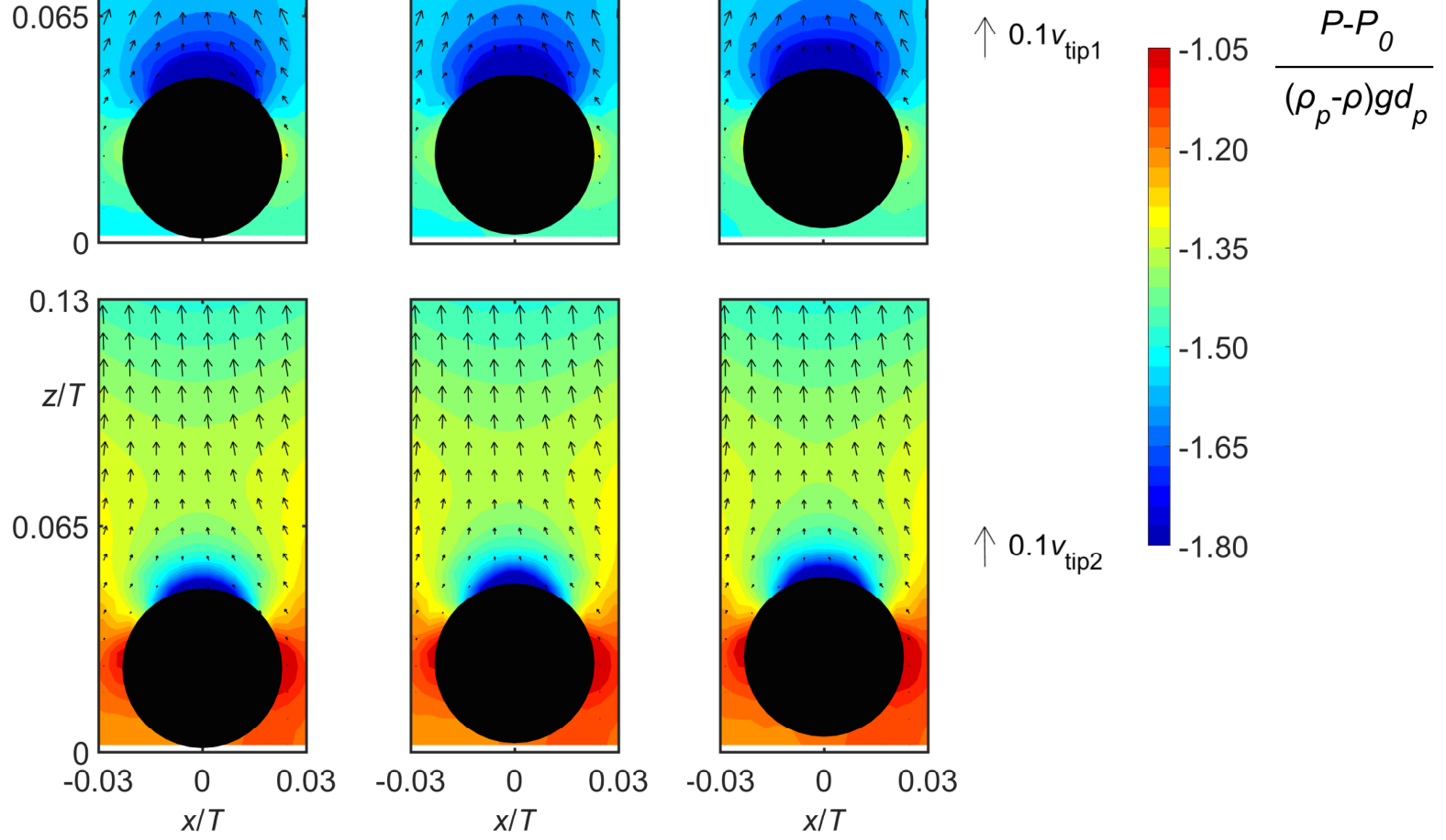

Figure 9. Dimensionless velocity vector and pressure difference around a particle with the Rushton turbine. Top row: $\operatorname{Re}=28.34$ and $\theta_{\text {sim }}=2.03$; bottom row: $\operatorname{Re}=8.14$ and $\theta_{\text {sim }}=1.22$. The spacing between the lowest position of the sphere and the bottom wall in the left, the middle, and the right columns are $0.36 \Delta, 0.72 \Delta$, and $1.2 \Delta$ respectively. Two tip speeds of the impeller $v_{\text {tip } 1}=1.70 \mathrm{~m} / \mathrm{s}$ and $v_{\text {tip } 2}=0.49 \mathrm{~m} / \mathrm{s}$ as indicated. 


\section{Figure 10}
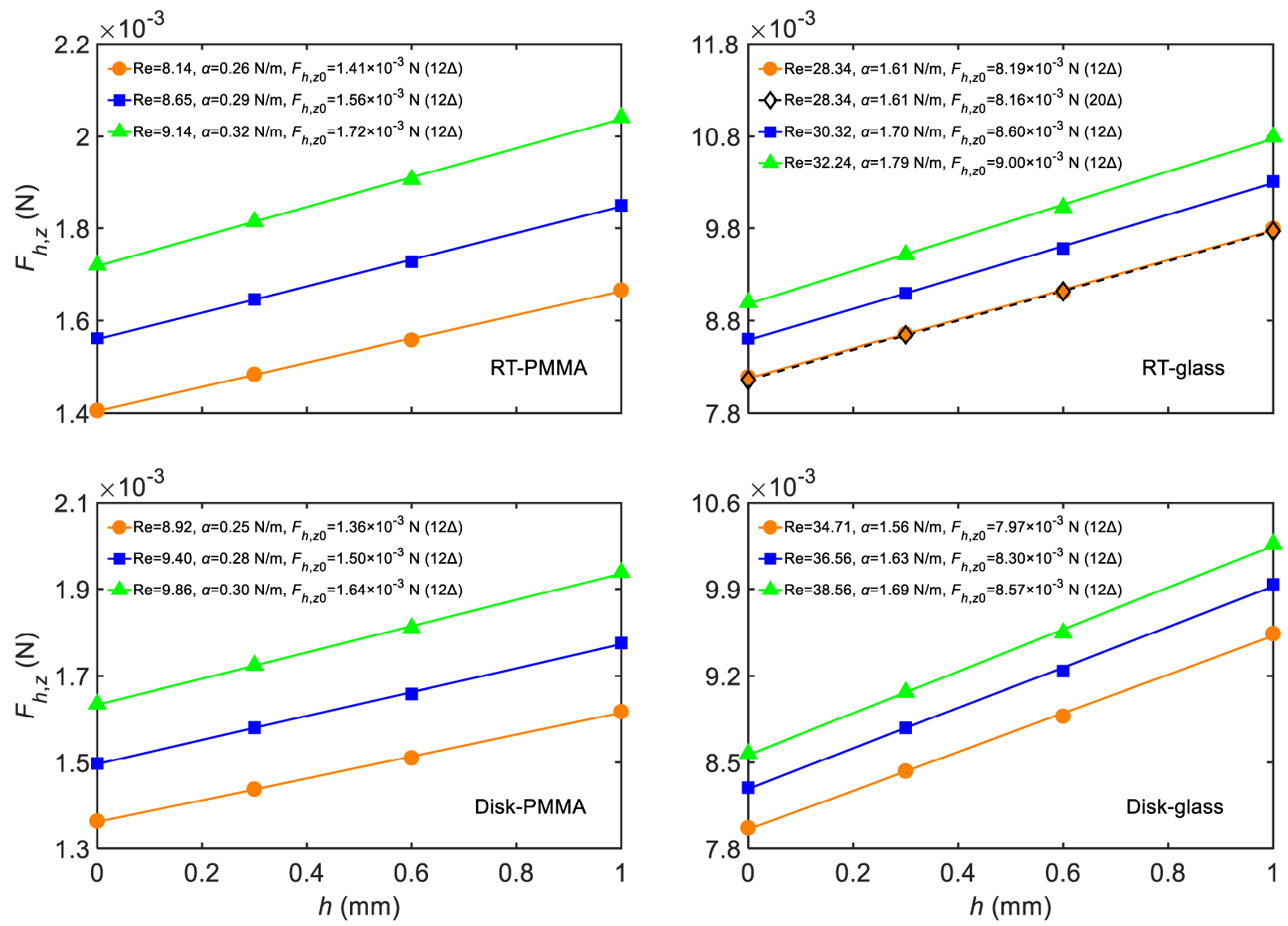

Figure 10. Symbols: simulated hydrodynamic force on a sphere as a function of height at the specified Reynolds number. Lines: linear fit of the symbols with intercept $F_{h, z 0}$ and slope $\alpha$.

The resolution of each simulation is indicated by the number of lattice spacings $\Delta$ per particle diameter. 


\section{Figure 11}
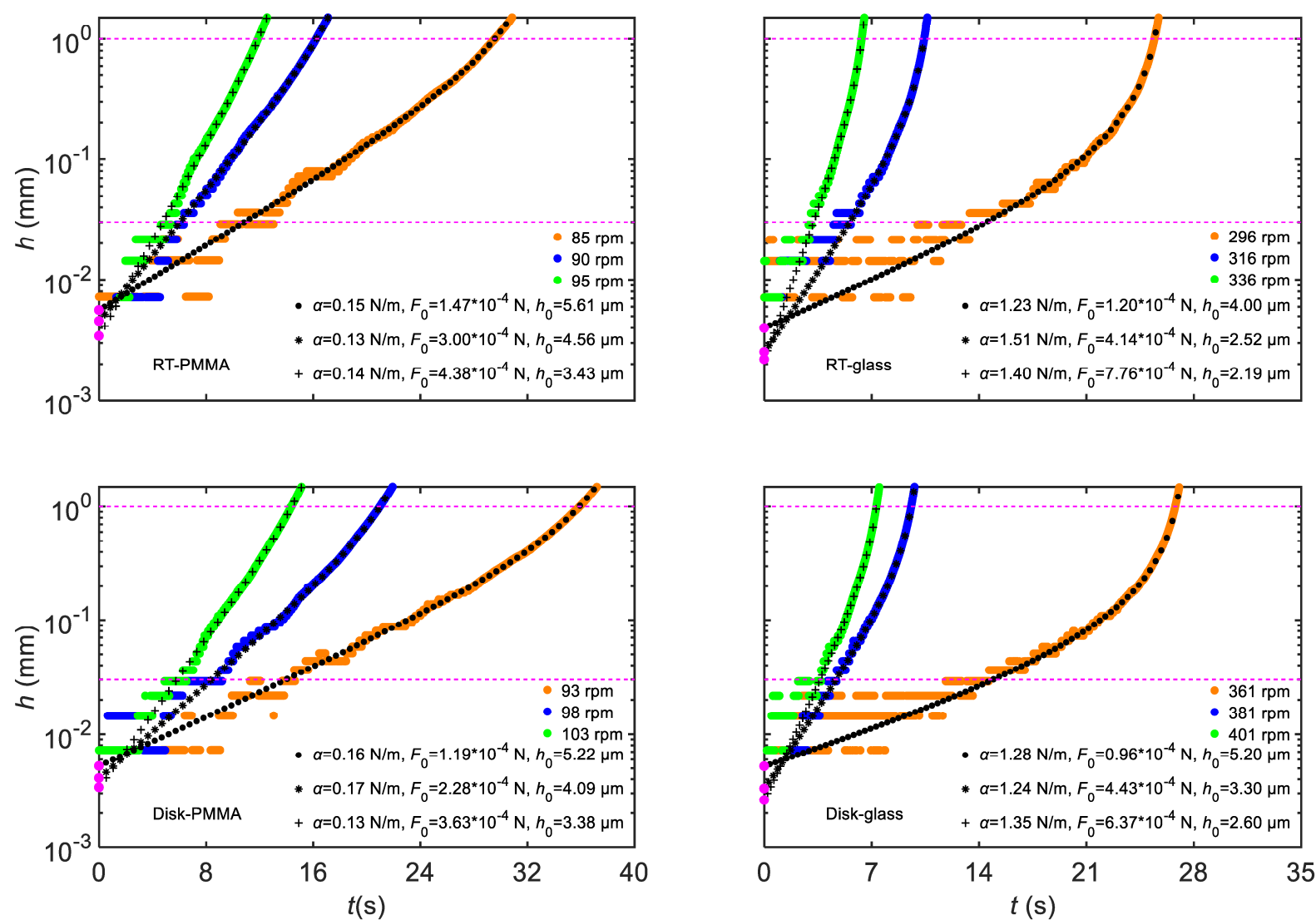

Figure 11. The spacing of the particle as a function of time with a logarithmic scale on the vertical axis, where $\alpha, F_{0}$, and $h_{0}$ are fitted by Eq. 2 . The data (from $h=0.03 \mathrm{~mm}$ to 1.0 $\mathrm{mm}$ ) between two pink dashed lines are used to perform the nonlinear fitting. The pink point represents $h_{0}$. 


\section{Figure 12}

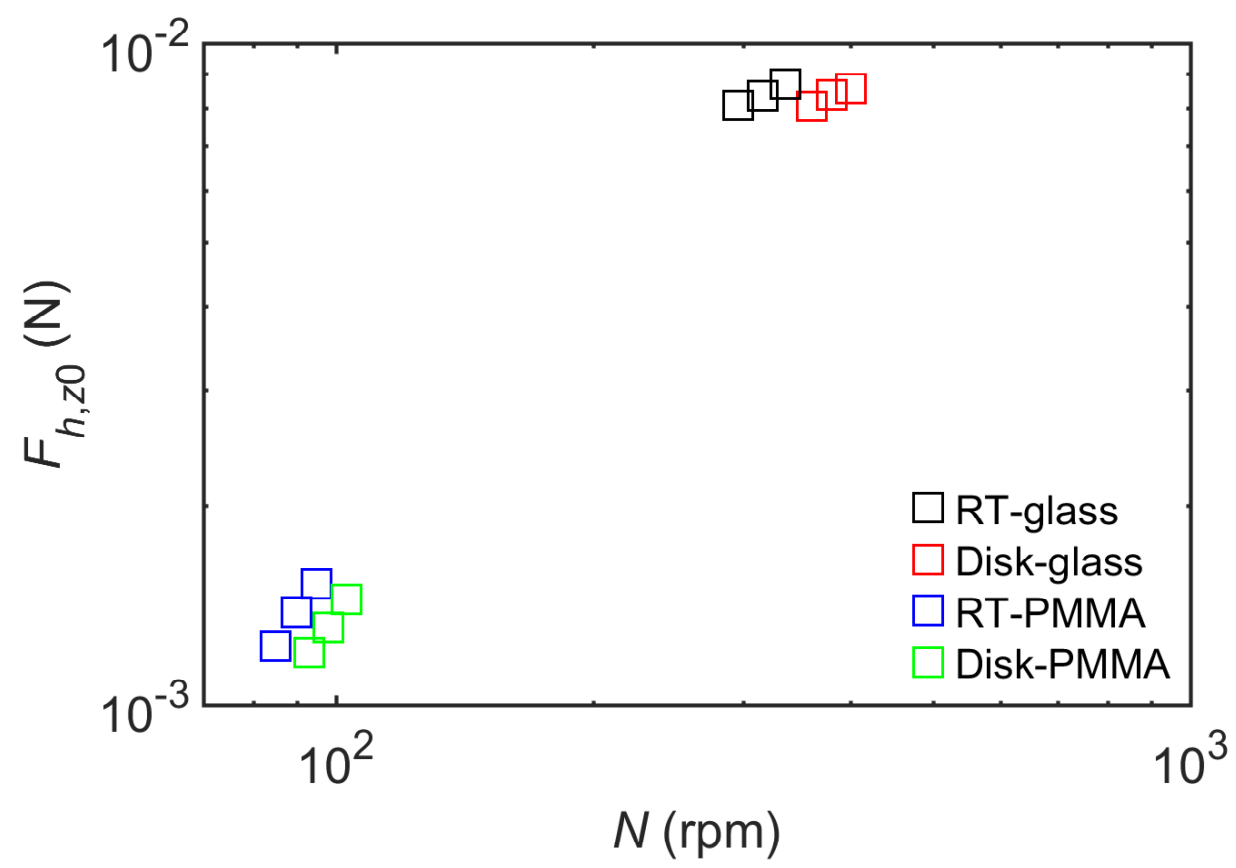

Figure 12. Vertical hydrodynamic force $F_{h, z 0}$ as a function of impeller speed $N$ as obtained through fitting height versus time. Black symbols: RT-glass; red symbols: Disk-glass; blue: RT-PMMA; green: Disk-PMMA. 


\section{Table 1}

Table 1. Experimental parameters and results for the lift-off impeller speed $N_{L O}$ and the impeller speed which is larger than $N_{L O}$.

\begin{tabular}{clccc}
\hline Combination & $N[\mathrm{rpm}]$ & $\operatorname{Re}[-]$ & $\theta_{\text {exp }}[-]$ & $t_{0.1} *[\mathrm{~s}]$ \\
\hline RT-glass & $296\left(N_{L O}\right)$ & 28.34 & 1.90 & 25.45 \\
& 316 & 30.32 & 2.17 & 10.44 \\
& 336 & 32.24 & 2.45 & 6.36 \\
RT-PMMA & $85\left(N_{L O}\right)$ & 8.14 & 1.14 & 29.60 \\
& 90 & 8.65 & 1.28 & 16.24 \\
& 95 & 9.14 & 1.43 & 11.90 \\
Disk-glass & $361\left(N_{L O}\right)$ & 34.71 & 2.83 & 26.81 \\
& 381 & 36.56 & 3.15 & 9.59 \\
& 401 & 38.56 & 3.49 & 7.32 \\
Disk-PMMA & $93\left(N_{L O}\right)$ & 8.92 & 1.37 & 35.92 \\
& 98 & 9.40 & 1.52 & 21.01 \\
& 103 & 9.86 & 1.68 & 14.39 \\
\hline
\end{tabular}

* After the target impeller speed $N$ is achieved, $t_{0.1}$ is defined as the moment that the particle is lifted off by $1 \mathrm{~mm}$ (which is $0.1 d_{p}$ ). 


\section{Table 2}

Table 2. Parameters and results used in experiments, fitting process, and simulations.

\begin{tabular}{|c|c|c|c|c|c|c|c|c|c|c|c|c|c|}
\hline Combination & $N$ [rpm] & $\operatorname{Re}[-]$ & $\theta_{\exp }[-]$ & $\begin{array}{c}G_{\text {net }} \\
{\left[10^{-4} \mathrm{~N}\right]}\end{array}$ & $\begin{array}{c}h_{0, \mathrm{Eq} .1} * \\
{[\mu \mathrm{m}]}\end{array}$ & $\begin{array}{c}h_{0}^{*} \\
{[\mu \mathrm{m}]}\end{array}$ & $\begin{array}{l}\alpha \text { (fit) } \\
{[\mathrm{N} / \mathrm{m}]}\end{array}$ & $\begin{array}{l}F_{0}(\text { fit }) \\
{\left[10^{-4} \mathrm{~N}\right]}\end{array}$ & $\begin{array}{l}F_{h, z 0}(\mathrm{fit}) \\
{\left[10^{-4} \mathrm{~N}\right]}\end{array}$ & $\theta_{\text {sim }} *[-]$ & $\begin{array}{l}\alpha(\operatorname{sim}) \\
{[\mathrm{N} / \mathrm{m}]}\end{array}$ & $\begin{array}{l}F_{0}(\operatorname{sim}) \\
{\left[10^{-4} \mathrm{~N}\right]}\end{array}$ & $\begin{array}{c}F_{h, z 0}(\operatorname{sim}) \\
{\left[10^{-4} \mathrm{~N}\right]}\end{array}$ \\
\hline \multirow[t]{4}{*}{ RT-glass } & 296 & 28.34 & 1.90 & 79.26 & 2.39 & 4.00 & 1.23 & 1.20 & 80.46 & 2.03 & 1.61 & 2.60 & 81.86 \\
\hline & $296^{*}$ & 28.34 & 1.90 & & 2.39 & 4.00 & 1.23 & 1.20 & 80.46 & 2.03 & 1.61 & 2.30 & 81.56 \\
\hline & 316 & 30.32 & 2.17 & & 2.59 & 2.52 & 1.51 & 4.14 & 83.40 & 2.32 & 1.70 & 6.74 & 86.00 \\
\hline & 336 & 32.24 & 2.45 & & 2.46 & 2.19 & 1.40 & 7.76 & 87.02 & 2.62 & 1.79 & 10.73 & 89.99 \\
\hline \multirow[t]{3}{*}{ RT-PMMA } & 85 & 8.14 & 1.14 & 10.83 & 3.75 & 5.61 & 0.15 & 1.47 & 12.30 & 1.22 & 0.26 & 3.22 & 14.05 \\
\hline & 90 & 8.65 & 1.28 & & 3.38 & 4.56 & 0.13 & 3.00 & 13.83 & 1.37 & 0.29 & 4.78 & 15.61 \\
\hline & 95 & 9.14 & 1.43 & & 2.47 & 3.43 & 0.14 & 4.38 & 15.21 & 1.53 & 0.32 & 6.37 & 17.20 \\
\hline \multirow[t]{3}{*}{ Disk-glass } & 361 & 34.71 & 2.83 & 79.26 & 2.23 & 5.20 & 1.28 & 0.96 & 80.22 & 3.03 & 1.56 & 0.43 & 79.69 \\
\hline & 381 & 36.56 & 3.15 & & 2.50 & 3.30 & 1.24 & 4.43 & 83.69 & 3.37 & 1.63 & 3.72 & 82.98 \\
\hline & 401 & 38.56 & 3.49 & & 2.64 & 2.60 & 1.35 & 6.37 & 85.63 & 3.73 & 1.69 & 6.45 & 85.71 \\
\hline \multirow[t]{3}{*}{ Disk-PMMA } & 93 & 8.92 & 1.37 & 10.83 & 3.14 & 5.22 & 0.16 & 1.19 & 12.02 & 1.46 & 0.25 & 2.81 & 13.64 \\
\hline & 98 & 9.40 & 1.52 & & 2.91 & 4.09 & 0.17 & 2.28 & 13.11 & 1.63 & 0.28 & 4.15 & 14.98 \\
\hline & 103 & 9.86 & 1.68 & & 2.29 & 3.38 & 0.13 & 3.63 & 14.46 & 1.80 & 0.30 & 5.52 & 16.35 \\
\hline
\end{tabular}

* The finer resolution of 20 grid spacings over a sphere diameter is used in the simulations of the fixed glass particle with $\mathrm{Re}=28.34$

The value for $h_{0, \text { Eq. } 1}$ is estimated by Eq. 1

The value for $h_{0}$ is nonlinearly fitted by Eq. 2

$\theta_{\text {sim }}=1.07 \theta_{\text {exp }}$ 